\title{
RETRACTED ARTICLE: Ensemble of transfer learnt classifiers for recognition of cardiovascular tissues from histological images
}

\author{
Shubham Mittal ${ }^{1}$ [D
}

Received: 29 October 2018 / Accepted: 5 October 2019 / Published online: 24 October 2019

(c) Australasian College of Physical Scientists and Engineers in Medicine 2019

The author is retracting this article [1] because after publication he became aware that the exact source from where the models' ImageNet accuracy values were obtained could not be found and were not cited appropriately. Therefore the results (especially the data presented in Table 3) were not reported correctly. The Editor-in-Chief has invited the author to submit a new manuscript which will undergo peer review.

\section{Reference}

1. Mittal S (2019) Ensemble of transfer learnt classifiers for recognition of cardiovascular tissues from histological images. Phys Eng Sci Med. https://doi.org/10.1007/s13246-019-00807-9

Electronic supplementary material The online version of this article (doi:https://doi.org/10.1007/s13246-019-00807-9) contains supplementary material, which is available to authorized users.

Shubham Mittal

shubhammittl@gmail.com

1 Department of Electronics and Communication Engineering, Ambedkar Institute of Advanced Communication

Technologies and Research, Delhi, India 